\title{
Undergraduate Evo-Devo Laboratory Exercise: Butterflies Causing Near- Graduate Research Experience
}

\author{
Tomislav Terzin \\ Department of Science, Augustana Faculty, \\ University of Alberta, Camrose, Alberta, Canada
}

\begin{abstract}
The goal of lab exercise described in this paper is to provide biology undergraduate students with research experience comparable to graduate studies but performed during regular lab sessions. Working on regular weekly 3 hour lab projects, students dedicate a portion of time to do continuous experiments on butterfly wing pattern formation. In that way students are capable to generate experimental results superior in comparison to regular lab projects. Procedure which is stretched across several weeks includes observation of butterfly growth and metamorphosis, cold shock experiments and pupae injections with sodium tungstate, chemical which alters wild type colour patterning through the modification of cell signals. Furthermore students learn how to spread specimens, photograph them, and do comparative analysis of wild type and altered colour morphs of Painted Lady with other butterfly species from the same genus. In that way students compare induced polyphenism with the actual evolution of colour patterns, getting familiar with evodevo paradigm.
\end{abstract}

\section{Introduction}

Easy rearing, rapid growth and complete metamorphosis of worm-like caterpillar into colorful winged butterfly, makes it attractive object for K-12 science projects. At the university level however, butterflies are not often seen as a model system in undergraduate biology labs. What is suitable for elementary school may look "too simple" for the university. But appearance may be deceiving. Starting in mid 90', several researchers recognized the great potential butterflies have as model organisms in developmental biology and evolution of development "evo-devo" [1] [3]. Two dimensional wing surface decorated with complex colour patterns made by rows of microscopic pigmented scales, make butterflies perfect models for the study of pattern formation [2] [7]. This spectacular advancement calls for additions in our undergraduate lab practices. Here I represent a 300 level undergraduate developmental biology lab which uses Painted Lady butterflies to teach students one of the most advanced chapters of developmental biology: evolution of development or evo-devo.

\section{Materials and Methods}

Painted Lady caterpillars (Vanessa cardui) of $2^{\text {nd }}$ or $3^{\text {rd }}$ instar were obtained from Boreal Science Catalogue \# 6700700 for the Fall lab and from Merlan Scientific who is the distributor for Carolina Biological. The catalogue number is RE- 144070 . Although $V$. cardui is a cosmopolitan species native to $\mathrm{N}$. America and can be imported from USA to Canada and vice versa without any wildlife permit, customs personnel sometimes is not well informed about regulations and can make delays. To avoid complications with the import of time sensitive live organisms, the best is to order them from a supplier within the country. Artificial food was supplied along with the caterpillars. Tools and supplies for spreading butterflies were obtained from Bioquip (catalogue numbers are provided and quantity should be adjusted to the number of students): 4747 butterfly forceps; 1134R pinning strip; 1146S four-hole pin holder; 1208S2 bioquip insect pins no.2 stainless 100/pack; 1206 glass headed pins pack 100; 1021 Adjustable spreading board; $1154 \mathrm{E}$ pigma pen black $0.20 \mathrm{~mm}$; 1213 blank labels 11/16 x 5/16" and 1002F box with foam pinning bottom. Sodium tungstate dehydrate was obtained from SIGMA T2629-100G.

\subsection{First week - rearing}

Caterpillars were supplied as a whole brood in a deli-style container with artificial food (Fig.1A). Each student transferred 6 caterpillars individually into small desert cups using fine brush. Before transfer, about 1-1.5 cubic $\mathrm{cm}$ of artificial food was added in each desert cup using spatula. Several holes were 
punched in the lids and a piece of filter paper placed beneath the lid. Each student labelled 6 desert cups. It takes 5-9 days for the caterpillars to turn into pupae. Students were advised to check caterpillars every day and to take notes about any observed changes (the number of moltings, the speed of growth, the size increase, the rate of food consumption, possible contamination, formation of pupa etc.).

\subsection{Second week - pupation}

When the caterpillars have eaten about half the food, cleaning should be performed, by opening the lid and sweeping out the frass (caterpillar droppings that look like balls) and webbings with the small paintbrush. Paper under the lid should be replaced for humidity control. Upon pupation, two pupae were used as a wild type control and transferred in "wt" rearing cage at RT (Fig.1B). Another four pupae remaining in small desert cups but with removed remnants of food and frass, were exposed to $+4^{\circ} \mathrm{C}$ cold-shock [6] [12].

\subsection{Third week - injection}

Out of four cold-shock pupae, two should remain exposed to cold shock for two more weeks (21 days total) while another two should be injected with $3 \mu$ of $1 \mathrm{M}$ sodium tungstate and transferred in "injected" rearing cage on RT [10]. In their regular development, painted lady will need another 7-10 days to form adult body within the pupa, and to emerge as a butterfly. That process is postponed in cold-shock treatment. It is crucial to expose pupae to cold-shock within 3-8 hours after pupation [12]. The same is crucial for the success of injections but it can be postponed by keeping pupae several days on cold before injection is applied. This is very important step in order to be able to do injections during regular lab time but still have "early" pupae.

\subsection{Fourth week-spreading}

By this time, wt butterflies emerge and most likely injected butterflies emerge as well. About one day before butterflies emerge, wing colour patterns become visible under the pupal cuticle. It is important to allow butterflies to emerge and to spread and dry their wings uninterrupted. They have to have sufficient room to spread wings properly, for which rearing cage is optimal. Students will not be able to analyze wing patterns if wings are not developed properly. 12-24 hours after butterflies have emerged, students will take them out from the cage one by one, and anesthetize them with few drops of ethyl acetate for $10 \mathrm{~min}$ in a closed jar. Once butterflies are sacrificed students pin their thorax and spread them on the spreader following regular procedure for pinning and spreading butterflies. This requires some skill. Appropriate labels should be kept pinned near butterfly specimens on the spreader. At the end of lab, butterflies should be transferred on spreaders and put on $-20^{\circ} \mathrm{C}$ overnight. That will insure that no animal would recover from anesthesia and suffer being pinned alive. Next day spread butterflies should be taken from the freezer and left on RT to dry until next lab.

\subsection{Fifth week-imaging}

Both wt and injected dry butterfly specimens were taken from spreader and put into collection box or drawer with their labels pinned under each butterfly (Fig.2B). All four specimens (2 wt and 2 injected) were photographed on both dorsal and ventral side (Fig.2). Students transferred digital images from lab camera to individual memory sticks or took images using their own cameras. After 21 days, cold-shock treatment also should be put into rearing cage on RT and after next 7 days those butterflies should also emerge. Spreading and imaging procedure should be repeated on them as well (Fig.2 C and D).

\subsection{Seventh or eight week - data analysis}

This was the time for discussion and preparation for written assignment which was due 1-2 weeks after the last experimental procedure was performed on cold-shock group of butterflies.

\section{Results and Discussion}

It is well known that cold stress induces changes in colour pattern development in butterflies [4] [13]. However, if particular chemical agents like sodium tungstate are injected in a developing pupa, stronger and more frequent modification can be achieved [10]. Also see (Fig.3 and Tab.1). It is important that the injection takes place only within a narrow time frame of few hours. For Painted Lady optimal time is around 6 hours upon pupation for both cold shock exposure and sodium tungstate injections [10] [12]. Cell signaling responsible for species specific pattern formation occurs only briefly and the interference with natural cell signaling has to be achieved only at that 
time. Pattern modification cannot be achieved on old pupae. Because of that, it is important to be flexible in the lab design. Lab instructor has to be available and to monitor butterfly development outside of the regular lab schedule in order to capture that crucial period of development and place young pupae on $+4{ }^{\circ} \mathrm{C}$. The success of the entire project depended on that moment. Our lab practice proved that injection can be successfully postponed until next lab time, if the pupae were transferred on cold as young. First several hours after pupation can be determined by the colour of pupae which is greenish. As pupa matures and the cuticle hardens, the colour turns into yellowish-brown. At that point is usually too late to successfully apply cold shock or sodium tungstate treatment.

Sodium tungstate is an inhibitor of protein tyrosine phosphatase (PTPase) [5]. By inhibiting PTPases, tungstate prevents signals from being properly received by the pattern forming scale cells (Fig.2 E and F). Since the actual dose of tungstate received by cells and the precise timing of injection are impossible to reproduce exactly, the effect is variable but within the borders ranging from mild pattern alteration to very drastic ones (compare weak and strong phenotypes of the injected specimens in Fig.3). Strongly affected specimens tend to "erase" natural patterns (Fig.2 E and F compared to Fig.2 A and B). Cold shock usually produces less drastic effects (Fig.2 C and D), often with some small pattern elements missing on otherwise wild type like specimen. Melanisation often happens with cold shock specimens which tend to be darker than wt specimens (see strong cold shock phenotypes in Fig.3).

Beside personal results, or results obtained together with the lab partner, each student should also obtain the class data. Large sample helps students to see the regularity and predictability of developmental processes. Class data also help students to better understand the design of experiments and the purpose of controls and repeated treatments. It is recommended that the lab instructor provides a table, similar like Tab.1, which can be used to gather class data. There are several important conclusions which can be derived based on the observations and comparisons of class data. In the Tab.1 are provided results of two actual lab groups of Augustana students who conducted this exercise in 2012 (bold numbers) and 2013. There are two control groups of butterflies in this lab exercise. The first control group is "wild type" or not treated pupae. They develop regularly and we expect to see a high survival rate and no modification of colour patterns (Tab.1). The second control group are pupae injected with $3 \mu \mathrm{l}$ of sterile distilled water. The purpose of this control is to reveal the effect of injection alone as an invasive experimental technique, to the survival of treated pupae. If significantly more pupae die in sodium tungstate injected group than in water injected group, that would be an indication of toxicity of applied amount of sodium tungstate. If similar rate of lethality is observed in both the control and the treatment group, that would indicate that the act of injection alone causes the death outcome, regardless of the content of the needle. Statistical analysis can be run to provide the information if there is significant difference between the treatments and the controls, but due to the overall complexity of this lab and the fact that students got familiar with the statistical analysis in other labs within the same course, author did not insist on the statistical analysis, which however certainly can be included in this lab exercise. Another conclusion which can be derived from the observation of class data (see Tab.1) is that the lethality can be different in different lab groups, but usually matches between the injection control and the injection treatment within the same lab group. This may be caused by various factors, starting from the bacterial or viral contamination of equipment to the level of skill which may be different in different lab groups. Another important observation based on the numbers in the Table 1 . is the efficacy of treatments. It is safe to assume that over a half of cold shock treated and sodium tungstate treated pupae will survive and emerge as butterflies. It is also safe to assume that roughly a half of that half will be affected phenotypically and will express visible difference in wing colour patterns compared to the wild type control. That is the reason why so many caterpillars (at least six per student) are needed to be successful in getting results from this lab. Not all students will be able to produce expected results for all controls and treatments, but within the class, there will be enough examples to make at least a qualitative observation. Although there is no firm ethical regulation in place for work with the invertebrates, we need to recognise the value of invertebrate life in a same way as we recognise the value of vertebrate life. To reduce the number of sacrificed caterpillars, only several students in each lab group were assigned to do injection control experiment. Their results then were used by entire group.

Gathering the class data is crucial for the ability to observe the entire spectrum of possible phenotypic changes within and between the treatments (see Fig.3). It is possible to sort affected specimens in both cold 
shock and tungstate injection treatments, by the extent their colour patterns depart from the wild type. Roughly we can separate phenotypes in two groups: weak phenotypes which more resemble wild type and strong phenotypes which are seriously departed from the wild type colour scheme (Fig.3). Of course, this is an arbitrary division and we could also be able to introduce intermediate phenotypes in between these two categories, but the interpretation about where the intermediates start and where they end would be very difficult to justify.

It is important to explain to the students that what we here call "phenotypes" does not have anything to do with the genotype. All genotypes of treated butterflies are "wild type". There are no mutations induced by our treatments on the DNA level. What we do is the modification of cell signals which execute the genetic program of colour pattern formation [4] [6] [11] [14]. The result is an array of different colour patterns induced in the laboratory by the cold shock treatment or using the chemicals (sodium tungstate). An instance where the same species with the same genotype can express different colour forms is known as polyphenism. In the nature, polyphenism is usually triggered by different seasonal stimuli. The most studied example is European map butterfly Araschnia levana which has different colour forms in its spring and summer generation [14]. That is seasonal polyphenism. In this laboratory exercise we induce polyphenism in a species which naturally have uniform colour pattern and is monomorphic both seasonally and sexually.

There is a certain correlation between the phenotypic effects of cold shock exposure and tungstate injections with the evolution of pattern elements within the genus Vanessa [8] [9] [11]. Fig.4 represents various Vanessa species which as wild types do have pattern elements which resemble altered Vanessa cardui patterns. Compare second weak cold shock phenotype from the left in Fig.3 with Vanessa carye shown in Fig.4A. Also compare lower wings of the spread specimen in Fig.1D with the spots of the lower wings of Vanessa gonerilla represented in Fig.4E. Furthermore, melanistic strong cold shock phenotypes on Fig.3 can be compared with overall darker species Vanessa buana shown on Fig.4C and D. These are just some examples among many. Various weak and strong $V$. cardui phenotypes could be compared with over 20 existing Vanessa species, most of which are shown in the drawer on Fig.5 but not clearly visible. There are many similarities, but we need to keep in mind that these similarities are the subject of interpretation. The challenging part in the preparation for this laboratory exercise is to obtain a collection of various Vanessa species to be compared with $V$. cardui produced phenotypes. Unlike the Painted lady which has a cosmopolitan distribution, many species of the genus Vanessa are endemic to various geographic regions and hard to obtain. The easiest way to obtain at least several species is to search online stores of dry insect specimens listed on the front page of www.insectnet.com. Occasionally, some rare Vanessa species can also be found offered on eBay. Also, images of other species, obtained from the books, various collections or internet, could be sufficient to provide meaningful comparisons if the copyright issues can be solved. If nothing else, three Vanessa species represented here in Fig.4 can be of some use. Alternatively, this lab in its less complex form, can be offered based on the study of $V$. cardui only, without any evolutionary comparison with other species, but still within the theoretical framework of colour pattern formation.

\section{Conclusions}

If we closely examine Figure 2 and particularly Figure 3, we can derive several general conclusions about the nature of colour pattern changes that we were able to induce:

a) Both cold shock and sodium tungstate treatments are capable to induce changes in colour pattern formation in Painted Lady butterfly.

b) These changes are similar but not identical between cold shock and sodium tungstate treatments.

c) Sodium tungstate induces stronger and more frequent changes than cold shock treatment.

d) Induced polyphenism affects both dorsal and ventral surfaces of all four wings.

e) Colour changes are variable but follow a relatively uniform and predictable sequence based on the intensity of achieved modification.

f) Weak phenotypes in both cold shock and sodium tungstate treatments tend to have lighter background surface of ventral side of lower wings than the wild type.

g) Strong phenotypes in both cold shock and sodium tungstate treatments tend to have darker background surface of ventral side of lower wings than the wild type.

h) Features described under f) and g) can help us to easily identify and score the phenotypes while butterflies are in the rearing cage with their wings 
closed in natural position above the body with the exposed ventral wing surface.

i) Wild type pattern elements tend to blend or to get erased as we progress from weak toward strong phenotypes.

j) Submarginal dark spots on the dorsal surface of lower wings are the best signifier which marks the transition from weak toward strong phenotypes in sodium tungstate treated specimens. Wild type and weak phenotypes have dark brown submarginal spots which then turn bluish or dark brown with white spot in the centre. In strong sodium tungstate induced phenotypes entire spots become white or yellowish and finally disappear in strongest phenotypes. At the same time, this feature marks the most prominent difference between cold shock and sodium tungstate treatments since it is absent or extremely seldom seen in cold shock phenotypes. Students appreciate the opportunity to take part in the complex experimental procedure which enriches them with new, almost artistic skills while at the same time they embark on the cutting edge of evo-devo science. They managed to visualise science on the wings of butterflies. That is the experience which they will remember and treasure for a long time (Fig.5).

\section{References}

[1] Beldade, P., and P. M. Brakefield. 2002. The genetics and evo-devo of butterfly wing patterns. Nature Genetics. 3, pp. 442-452.

[2] Brakefield PM, French V. 1999. Butterfly wings: the evolution of development of colour patterns. BioEssays. 21, pp. 391-401.

[3] Carroll SB, Gates J, Keys DN, Paddock SW, Panganiban GE, Selegue JE, Williams JA. 1994. Pattern formation and eyespot determination in butterfly wings. Science 265(5168), pp. 109-14

[4] Mahdi SHA, Gima S, Tomita Y, Yamasaki H, Otaki JM. 2010. Physiological characterization of the coldshock-induced humoral factor for wing color-pattern changes in butterflies. J Insect Physiol. 56, pp. 10221031.

[5] Neel BG, Tonks NK. 1997. Protein tyrosine phosphatases in signal transduction. Curr Opin Cell Biol. 9(2), pp. 193-204.
[6] Nijhout HF. 1984. Colour pattern modification by coldshock in Lepidoptera. Journal of Embryology and Experimental Morphology 81(1), pp. 287-305.

[7] Nijhout HF. 1991. The Development and Evolution of Butterfly Wing Patterns. Washington (D.C.): Smithsonian Institution Press. Chapter 1, The Material Basis of Wing Color Patterns, pp. 1-22.

[8] Otaki JM, Yamamoto H. 2004a. Species-specific colorpattern modifications of butterfly wings. Develop. Growth Differ. 46, pp. 1-14.

[9] Otaki JM, Yamamoto H. 2004b. Color-pattern modifications and speciation in butterflies of the genus Vanessa and its related genera Cynthia and Bassaris. Zool Sci. 21(9), pp. 967-976.

[10] Otaki JM, Ogasawara T, Yamamoto H. 2005. Tungstate-Induced Color-Pattern Modifications of Butterfly Wings are Independent of Stress Response and Ecdysteroid Effect. Zool Sci. 22, pp. 635-644.

[11] Otaki JM. 2007. Stress-Induced Color-Pattern Modifications and Evolution of the Painted Lady Butterflies Vanessa cardui and Vanessa kershawi. Zoological Science. 24(8), pp. 811-819.

[12] Otaki JM. 2008. Physiologically induced color-pattern changes in butterfly wings: Mechanistic and evolutionary implication. Journal of Insect Physiology. 54, pp. 1099-1112.

[13] Stevens DJ. 2004. Pupal development temperature alters adult phenotype in the speckled wood butterfly, Pararge aegeria. Journal of Thermal Biology. 29 (4-5), pp. 205-210.

[14] Fric Z. and Konvicka M. 2002. Generations of the polyphonic butterfly Araschnia levana differ in body design. Evolutionary Ecology Research. 4, pp. 1017-1032.

\section{Acknowledgement}

I would like to thank biology technicians at Augustana campus: Rita Torok-Both and Marian Forre for their assistance in the preparation and organisation of this lab. I thank Augustana communications specialist Christopher Thrall for taking group picture in the butterfly lab and for collecting all student consents for public use of that picture. Last but not least, I thank all our biology students who have taken developmental biology course and participated in this lab in the last three years. This lab was designed for them and improved through the interactions with them. 


\section{Figures}
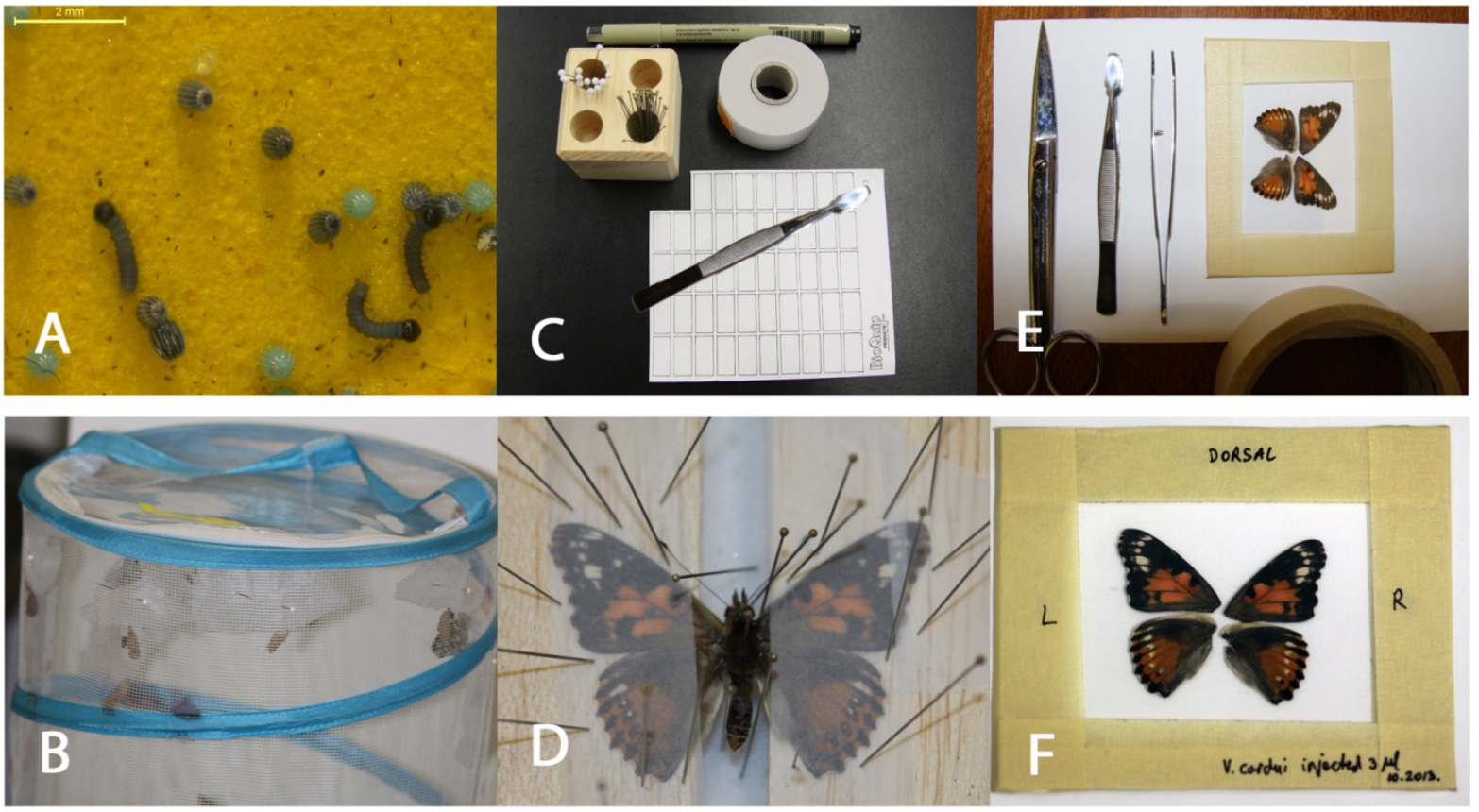

Figure 1. Rearing Vanessa cardui and two alternative ways of spreading specimens for the purpose of pattern analysis. Panel A first instar caterpillars emerged from eggs. Panel $B$ after pupation specimens were transferred to the rearing cage. Each pupa is hanging on the paper transferred from the desert cup and secured with a pin. On the right is visible one emerged butterfly; Panel $C$ tools needed for butterfly spreading. Killing jar and spreader are not represented. Spreaders are visible on Fig.4; Panel D fully spread butterfly supported by many pins.
Specimen itself is pierced only with a single pin through the thorax; Panels $E$ and $F$ represent alternative way of spreading wings. After specimen is sacrificed in killing jar, wings are cut in their base and positioned between two large microscope slides. Edges are connected with a duct tape. This is simpler and faster way to prepare specimens for imaging. The downside is that specimen is actually destroyed and only wings preserved. For authors knowledge, this is the first description of this methodology. 

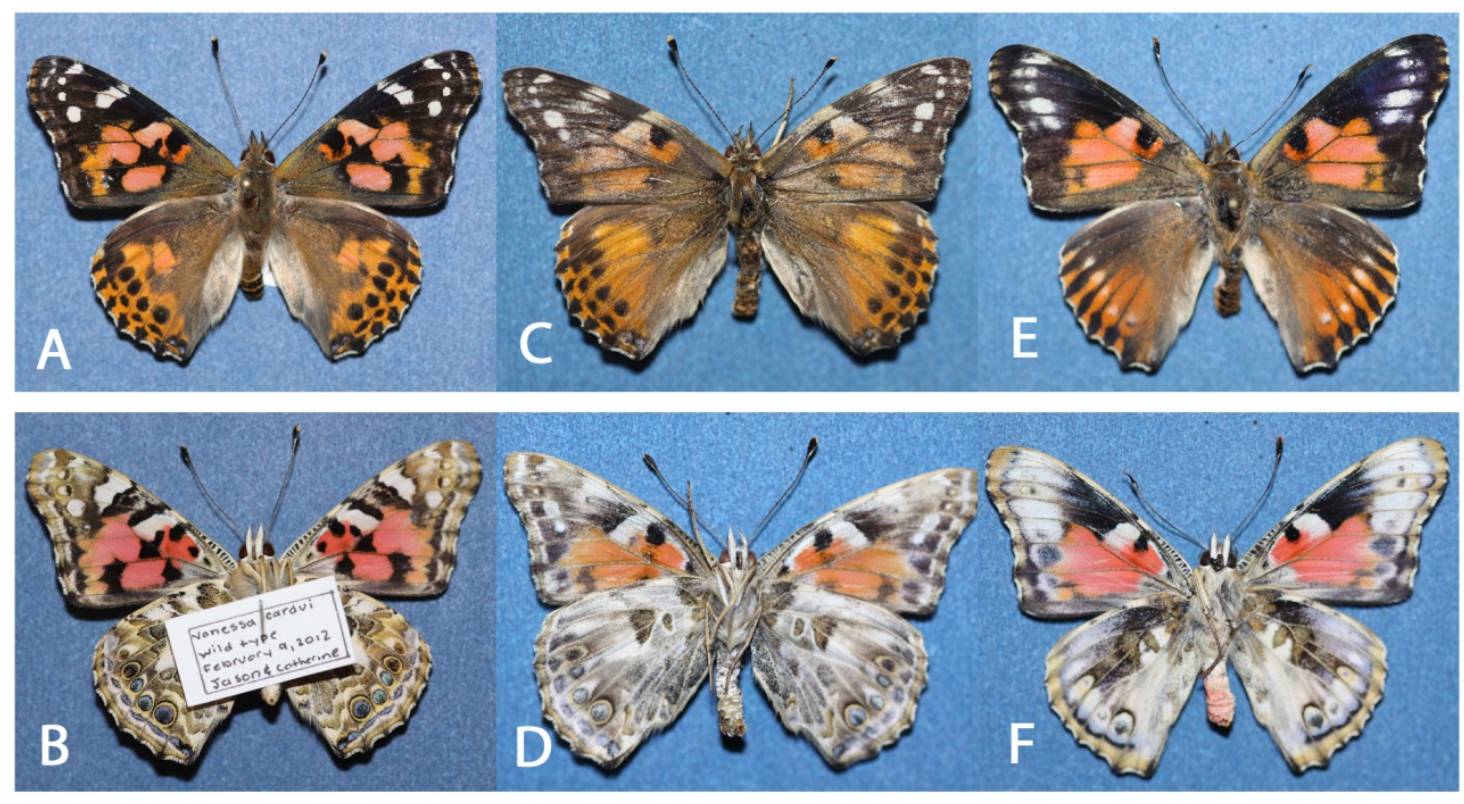

Figure 2. Vanessa cardui specimens produced in the lab. Vertical rows represent dorsal wing surface on the top and ventral wing surface of the same specimen on the bottom. From the left to the right:

Panels $A$ and $B$ wild type; Panels $C$ and $D$ young pupa was exposed to cold shock for 21 days; Panels $E$ and $F$ young pupa was injected with $3 \mu \mathrm{l}$ of $1 \mathrm{M}$ sodium tungstate. 


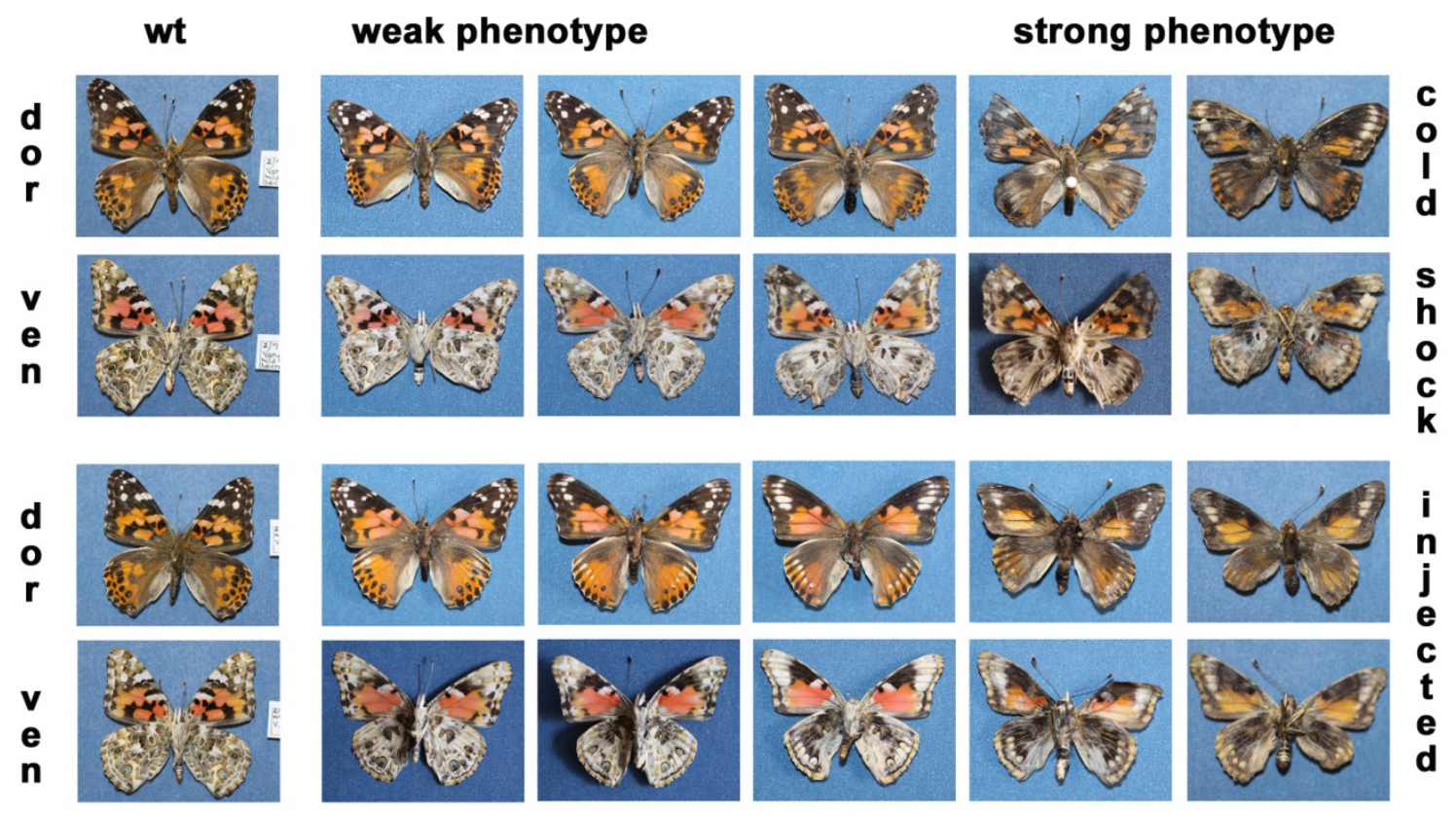

Figure 3. Vanessa cardui specimens produced in the lab. Represented is a large sample of phenotypes arranged from weak (on the left) to strong (on the right) for both cold shock and injected specimens and compared with the wild type non-treated specimens (on the far left). This type of succession is usually referred as "morphocline" [6]. Represented are both dorsal and ventral sides of specimens with the dorsal side on the top and the ventral side on the bottom for each specimen. Strong phenotypes are regularly coupled with the problems in pumping wings to their full size. Notice stronger effect of sodium tungstate injections compared to cold shock treatments. Injections also have different overall effect than cold shock but in both cases sequence of phenotypes can be followed uninterrupted from the weak ones toward the strong ones. 

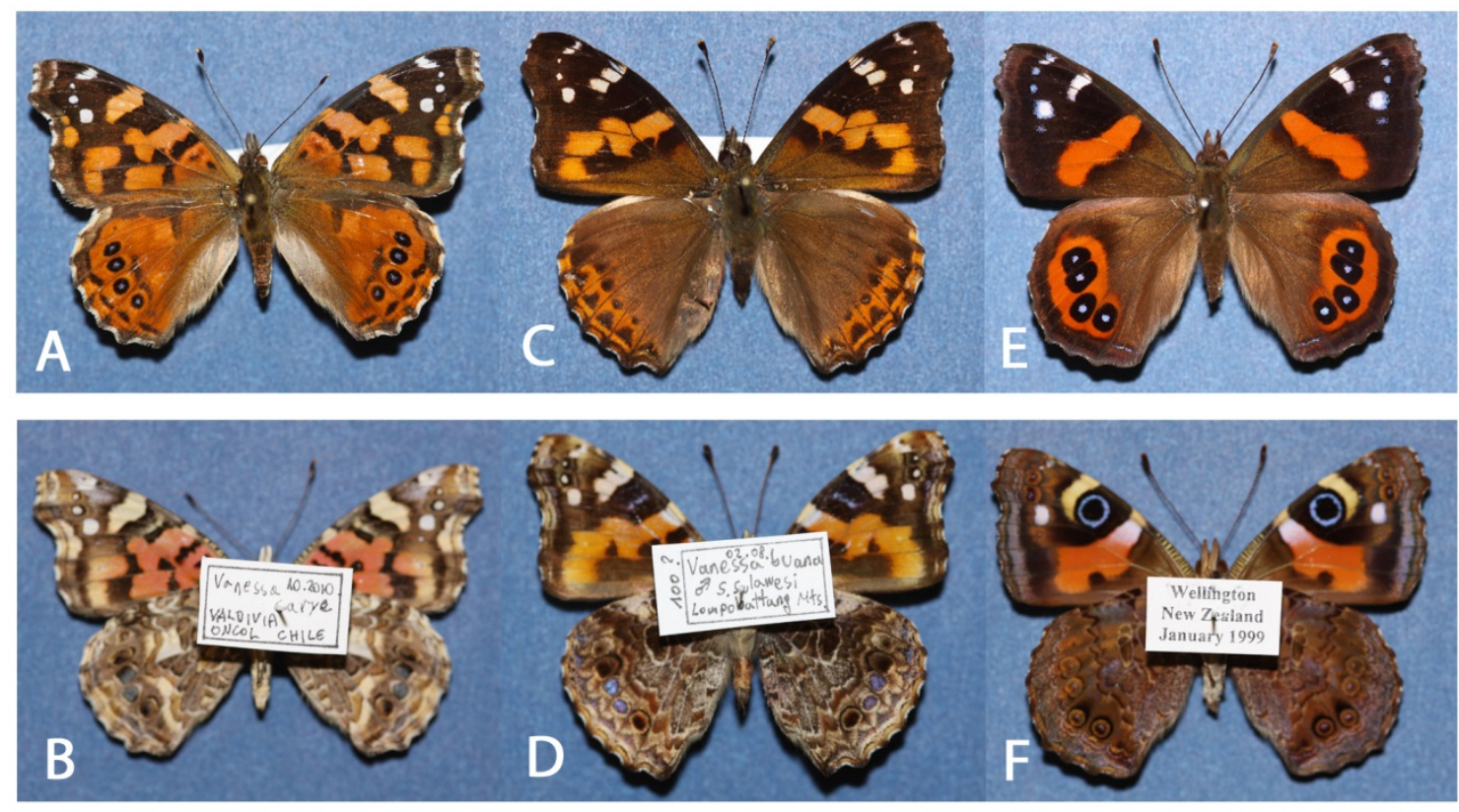

Figure 4. Three different species of genus Vanessa that we used to compare induced pattern changes in $V$. cardui to possible evolution of pattern elements within the genus. Vertical rows represent dorsal wing surface on the top and ventral wing surface of the same specimen on the bottom. From the left to the right: Panels A and B V. carye from Chile; Panels C and D V. buana from Sulawesi; Panels E and F V.gonerilla from New Zealand. Specimens are from authors collection.

\begin{tabular}{|c|c|c|c|}
\hline \multirow{2}{*}{$\begin{array}{c}\text { Treatment }(\mathbf{1 5 1}, \mathbf{1 4 4}) \\
\text { (total number of pupae) }\end{array}$} & \multicolumn{2}{|c|}{ Number of emerged butterflies } & $\begin{array}{c}\text { Not emerged } \\
\text { from pupae }\end{array}$ \\
\cline { 2 - 3 } & Affected & Not Affected & \\
\hline Wild-type $(\mathbf{4 4}, 36)$ & $\mathbf{0 , 0}$ & $\mathbf{4 2 , 3 5}$ & $\mathbf{2}, 1$ \\
Cold-shock $(\mathbf{4 9}, 41)$ & $\mathbf{7 , 8}$ & $\mathbf{2 0 , 1 9}$ & $\mathbf{2 2 , 1 4}$ \\
H2O inject. control $(\mathbf{2 0}, \mathbf{2 4 )}$ & $\mathbf{0 , 0}$ & $\mathbf{9 , 1 7}$ & $\mathbf{1 1}, 7$ \\
Injected tungstate $(\mathbf{3 8}, 43)$ & $\mathbf{1 0 , 1 5}$ & $\mathbf{1 1}, 20$ & $\mathbf{1 7 , 8}$ \\
\hline
\end{tabular}

Table 1. Two parallel examples of typical class results. The numbers of the first example are in bold for easier following and comparison. Represented is the number of butterflies in each treatment that were and were not able to emerge. Butterflies with the phenotype not different from the wild type were considered not affected and affected ones combine both weak and strong phenotypes. Both cold shock and injection treatments caused high but variable lethality of treated pupae (not emerged). 


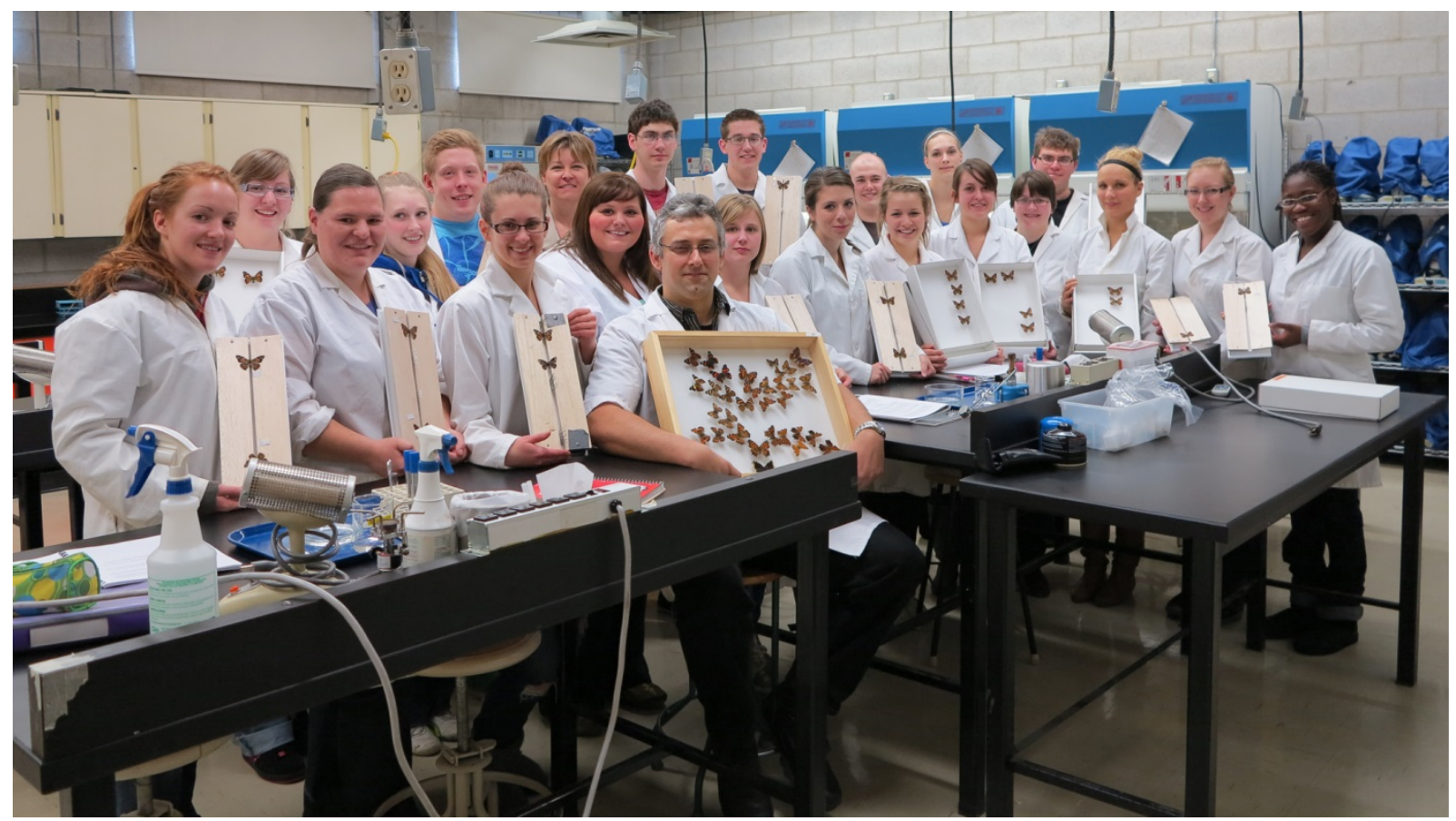

Figure 5. Third year developmental biology students at Augustana (Winter 2012) show their newly acquired skill in butterfly spreading. Injected and coldshock $V$. cardui specimens were compared with wt as well as with different species of Vanessa genus shown in the top half of the large drawer in the center of picture held by the author. In the back row on the left from the author is lab technician Rita Torok-Both who organised all technical details of butterfly lab. 